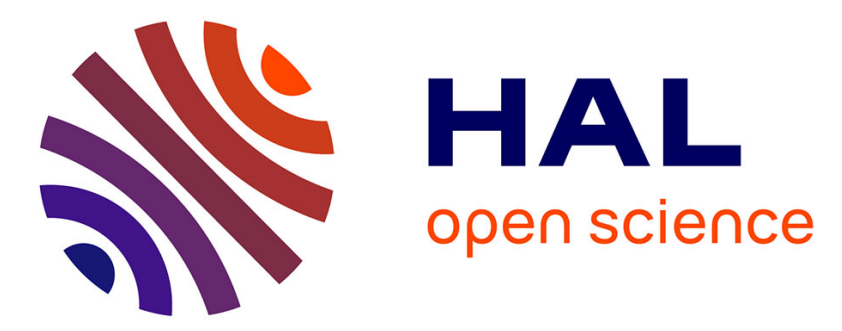

\title{
Pinching the cortex of live cells reveals thickness instabilities caused by myosin II motors
}

\author{
Valentin Laplaud, Nicolas Levernier, Judith Pineau, Mabel San, Lucie \\ Barbier, Pablo J Sáez, Ana-Maria Lennon-Duménil, Pablo Vargas, Karsten \\ Kruse, Olivia Du Roure, et al.
}

\section{To cite this version:}

Valentin Laplaud, Nicolas Levernier, Judith Pineau, Mabel San, Lucie Barbier, et al.. Pinching the cortex of live cells reveals thickness instabilities caused by myosin II motors. Science Advances, 2021. hal-03395597

\section{HAL Id: hal-03395597 \\ https://hal.science/hal-03395597}

Submitted on 22 Oct 2021

HAL is a multi-disciplinary open access archive for the deposit and dissemination of scientific research documents, whether they are published or not. The documents may come from teaching and research institutions in France or abroad, or from public or private research centers.
L'archive ouverte pluridisciplinaire HAL, est destinée au dépôt et à la diffusion de documents scientifiques de niveau recherche, publiés ou non, émanant des établissements d'enseignement et de recherche français ou étrangers, des laboratoires publics ou privés. 


\section{Pinching the cortex of live cells reveals thickness instabilities caused by myosin II motors}

\author{
Valentin Laplaud ${ }^{1,2}$, Nicolas Levernier ${ }^{3}$, Judith Pineau ${ }^{4}$, Mabel San Roman $^{4}$, Lucie Barbier ${ }^{2}$, \\ Pablo J. Sáez ${ }^{2}$, Ana-Maria Lennon-Duménil ${ }^{4}$, Pablo Vargas ${ }^{2}$, Karsten Kruse ${ }^{5}$, Olivia du Roure ${ }^{1 *}$, \\ Matthieu Piel ${ }^{2 *}$, Julien Heuvingh ${ }^{1 *}$
}

\begin{abstract}
The cell cortex is a contractile actin meshwork, which determines cell shape and is essential for cell mechanics, migration, and division. Because its thickness is below optical resolution, there is a tendency to consider the cortex as a thin uniform two-dimensional layer. Using two mutually attracted magnetic beads, one inside the cell and the other in the extracellular medium, we pinch the cortex of dendritic cells and provide an accurate and timeresolved measure of its thickness. Our observations draw a new picture of the cell cortex as a highly dynamic layer, harboring large fluctuations in its third dimension because of actomyosin contractility. We propose that the cortex dynamics might be responsible for the fast shape-changing capacity of highly contractile cells that use amoeboid-like migration.
\end{abstract}

Copyright @ 2021

The Authors, some rights reserved; exclusive licensee American Association for the Advancement of Science. No claim to original U.S. Government Works. Distributed under a Creative Commons Attribution NonCommercial License 4.0 (CC BY-NC).

\section{INTRODUCTION}

Dynamic cytoskeletal networks associated with the cell surface define the shape of mammalian cells $(1,2)$. In particular, the actin cortex, a thin network of actin filaments just beneath the plasma membrane, plays a central role in shaping the cell surface (3) and in defining its mechanical properties $(4,5)$. The actin cortex comprises, in addition to actin filaments, motors, membrane and actin linker proteins, actin nucleators (6) and cross-linkers, and regulatory proteins (7), which, together, render animal cell shape highly dynamic and able to adapt to external stimuli in a variety of physiological contexts such as cell migration or tissue morphogenesis.

Despite its central importance in cellular morphogenesis, the actin cortex remains poorly characterized (8). Its physical dimension (thickness) was only recently measured in cultured mammalian cells, using optical methods (9-11), but whether and to which degree this thickness varies in time is not known. So far, cell cortex mechanics has been probed through shallow indentation of the cell with an atomic force microscope (12) or through the twisting of ferromagnetic beads attached to the cell surface (13), but it is difficult in these experiments to separate the contribution of the cell cortex from the contribution of the rest of the cytoskeleton and cell organelles.

Since its discovery, there is a tendency to consider the cortex as a uniform two-dimensional (2D) structure, and its spatial and temporal heterogeneities remain largely unexplored $(4,8)$. This is, at least, partly due to experimental limitations in imaging a structure whose thickness is smaller than the optical resolution and also to the fact that contact probing can only be realized from the outside of the cell. In this work, we circumvent this obstacle by using a pair of probes (magnetic beads), one located inside the cell and the other on the outside. The attraction between the beads is controlled by an

\footnotetext{
${ }^{1}$ Physique et Mécanique des Milieux Hétérogènes, ESPCI Paris, PSL University, CNRS, Univ Paris, Sorbonne Université, Paris, France. ${ }^{2}$ Institut Curie and Institut Pierre Gilles de Gennes, PSL University, CNRS, Paris, France. ${ }^{3}$ Department of Theoretical Physics, University of Geneva, Geneva, Switzerland. ${ }^{4}$ Institut Curie, INSERM U932, PSL University, Paris, France. ${ }^{5}$ Departments of Biochemistry and Theoretical Physics and NCCR for Chemical Biology, University of Geneva, Geneva 1211 , Switzerland.

*Corresponding author. Email: olivia.duroure@espci.fr (O.d.R.); matthieu.piel@ curie.fr (M.P.); julien.heuvingh@espci.fr (J.H.)
}

external magnetic field, allowing us to slightly pinch the cortical layer. We can, in this manner, measure with unprecedented spatial accuracy and temporal resolution the thickness and dynamics of the cell cortex.

\section{RESULTS}

The magnetic pincher: A robust method to measure the physical properties of the cell cortex in live cells

Inspired by our previous work in which we probed thin layers of actin networks assembled in vitro between superparamagnetic beads $(14,15)$, we develop a new experimental setup in which two magnetic beads pinch the cell cortex, one being inside the cell and the other outside (Fig. 1A, beads 1 and 2). In the present work, we probe the cortex of primary bone marrow-derived dendritic cells from mice. These cells display amoeboid motility: a migration mode independent of focal adhesions, stress fibers, or large lamellipodial protrusions, which mostly rely on the fast remodeling of their actin cortex (16-19). Dendritic cells can also ingest large quantities of extracellular material including fluid (macropinocytosis) and particles (phagocytosis). This environment sampling activity allows them to take up antigens, which is the basis of their immune function and, in the present case, enables entry of large magnetic beads, independently of specific receptor engagement. Cells loaded with uptaken beads and mixed with freely floating beads are placed in an external homogeneous magnetic field. The field induces a tunable attractive force between the beads, which then pinch the cell cortex between them (Fig. 1B). Constant low force experiments provide thickness measurements, while varying the force gives access to mechanical properties. The experiment is facilitated by the spontaneous organization of magnetic beads into pairs or chains when exposed to a magnetic field. Transmission electron microscopy (TEM) imaging and fluorescent labeling confirm that ingested beads are surrounded by an endomembrane that has not fused with lysosomes (fig. S1, A to C) and that the cortex is pinched between the internal and external beads (Fig. 1B).

A first important point is to determine the accuracy of the measured distance between the beads. The beads are monitored in $3 \mathrm{D}$ at a frequency of $1.25 \mathrm{~Hz}$ over typically 5 to $15 \mathrm{~min}$ with transmitted 

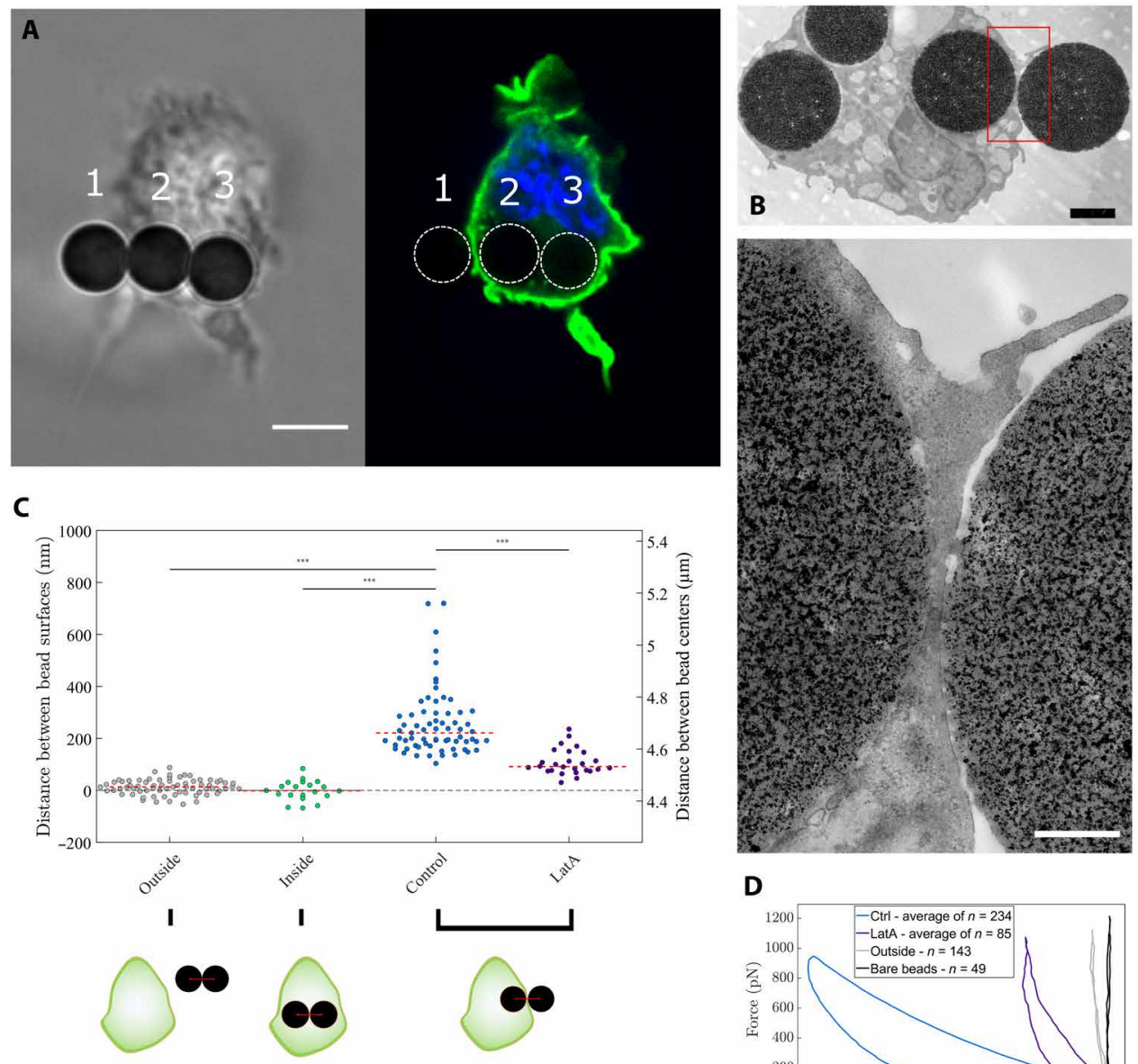

D

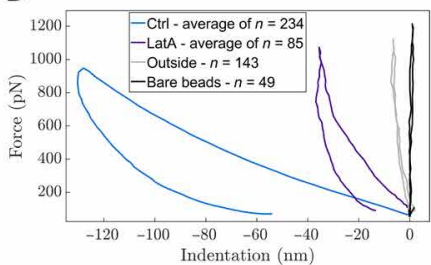

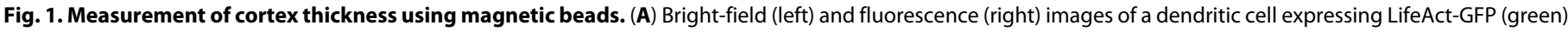

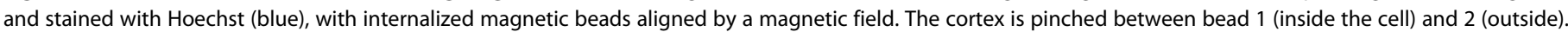

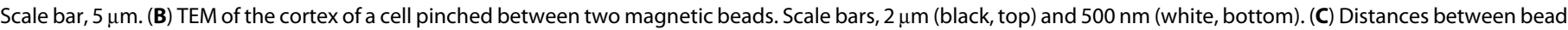

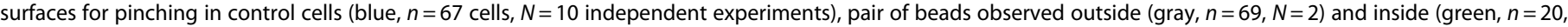

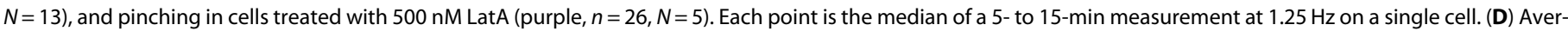

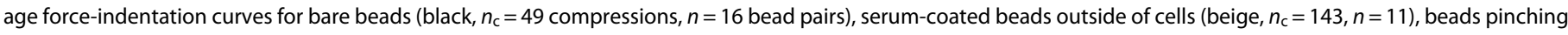

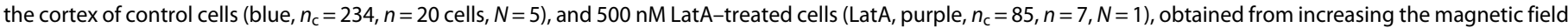
from $5 \mathrm{mT}(70 \mathrm{pN})$ to $50 \mathrm{mT}(\sim 1000 \mathrm{pN})$ in $1 \mathrm{~s}$ and decreasing it back.

light. The measurement accuracy on the distance between the center of two beads is approximately $2 \mathrm{~nm}(20)$ in the plane of observation and around $45 \mathrm{~nm}$ in the perpendicular direction (see the Supplementary Materials), resulting in an accuracy of $7 \mathrm{~nm}$ in the distance between bead centers. Subtraction of the bead diameter from the distance between the two centers gives the distance between the bead surfaces and thus the thickness of the pinched layer. We measure the beads to be highly monodispersed in size (fig. S1D), thus ensuring the precision of the distance between the bead surfaces. Overall, our measurement accuracy on the absolute thickness of the pinched layer is $31 \mathrm{~nm}$ (see the Supplementary Materials), which is $\sim 15 \%$ of the reported thickness of the cell cortex (9). In addition, the thickness variation in time can be determined with a much better accuracy $(7 \mathrm{~nm}$, as the uncertainty on the bead diameters does not enter into this calculation) at $>1 \mathrm{~Hz}$, more precisely than any method used so far to estimate cortical thickness.

\section{Dendritic cell cortical layer has a median thickness of $253 \mathbf{n m}$ and a Young's modulus of $7 \mathbf{~ k P a}$}

To determine the cortical layer thickness, a small magnetic field of $5 \mathrm{mT}$ is applied and produce a constant attractive force $(\sim 70 \mathrm{pN})$ between the beads. This force holds the beads in contact with the outer membrane and the inner face of the cell cortex so that the distance between the surfaces accurately reflects the cortex thickness. To estimate this thickness and control for potential artifacts, we perform three types of experiments:

1) We compare measurements of distances between two beads outside the cell, two beads inside the cell, and two beads pinching the cortex (Fig. 1C). While the distance between the surfaces of two beads inside or outside the cell is, on average, undistinguishable from zero, the distance measured for the cortical layer has a median value of $220 \mathrm{~nm}$ (Fig. 1C, blue). This measurement is consistent with measurements in Hela cells with subpixel-resolution fluorescence imaging (9). 
2) We increase the magnetic field, and thus the attraction force (up to $1 \mathrm{nN}$ ), to check whether the structure pinched between the beads is deformable (Fig. 1D). Increasing the force between beads gives relative measures, which are precise down to a few nanometers. This shows that bare beads are stiff and nondeformable (less than 1-nm indentation), and beads used in the live-cell experiment show a minimal indentation (below $10 \mathrm{~nm}$ ), which is due to the coating layer of serum (Fig. 1D, gray). In contrast, the cortical layer between a bead inside and a bead outside the cell is indented by more than $100 \mathrm{~nm}$ (Fig. 1D, blue), highlighting that a deformable object is being pinched. Upon force release, the distance between the beads relaxed, with a slight hysteresis, revealing the viscoelastic nature of the compressed material.

3) We compare control cells and cells in which the actin cortex has been disassembled using a high dose of latrunculin A (LatA) (Fig. 1, C and D). Treatment with $500 \mathrm{nM}$ LatA disassembles the actin cortex, as evidenced by LifeAct-GFP (green fluorescent protein) imaging and phalloidin staining in fixed cells (fig. S1, E and F). The remaining layer, measured at $92 \mathrm{~nm}$ (Fig. 1C, purple), which is significantly thinner than in untreated cells, is thicker than the distance between two beads inside the cell and can be reversibly indented by about $40 \mathrm{~nm}$ (Fig. 1D, purple). This indicates that there is still material pinched between the beads when cells are treated with LatA. It might contain a combination of membrane, polysaccharide chains such as glycocalyx, proteins that link the membrane to the actin cortex such as ezrin/radixin/moesin, and other cytoskeletal components such as septins or intermediate filaments $(2,21)$.

Increasing the magnitude of the magnetic field while monitoring the variation of the cortex thickness allows force-indentation curves to be extracted. On the range of deformation that we probed (up to $25 \%$ ), the mechanical response is linear (fig. S7E and Supplementary Materials) and the force indentation curves can be fitted with an appropriate model (22), taking into account the contact geometry of two beads deforming a thin layer (see the Supplementary Materials). The median of the estimated Young's modulus using the fit parameters is $7 \mathrm{kPa}$ for control cell cortices and $18 \mathrm{kPa}$ for the layer remaining after LatA treatment. The value of $7 \mathrm{kPa}$ is in accordance with measures of in vitro dense actin networks $(20,22,23)$ and compatible with the elastic modulus of whole cells (24).

The deformation of the cortex at low force in the constant field pincher experiments will induce a slight underestimation of the cortex thickness that we can calculate to be $13 \%$ using the value of $7 \mathrm{kPa}$ (see the Supplementary Materials). We thus estimate the median thickness of the undeformed cortical layer to be $253 \mathrm{~nm}$.

In conclusion, pinching the cortex with a pair of magnetic beads provides an accurate measure of the cell cortex thickness in live cells and a measure of its material properties. We evidence that dendritic cells have a thin and stiff cortical layer mostly composed of actin filaments, with properties comparable to the reported values in other cell types.

\section{The actin cortex thickness displays large nonperiodic local instabilities}

We next ask whether the cell cortex has a constant thickness, or whether thickness fluctuates in time. To address this question, we use timeresolved measures for single live cells comparing different bead configurations (Fig. 2A). Our measurement is extremely steady for beads outside the cells (control) and shows moderate fluctuations for inside beads, compatible with cell internal activity (25). In contrast,

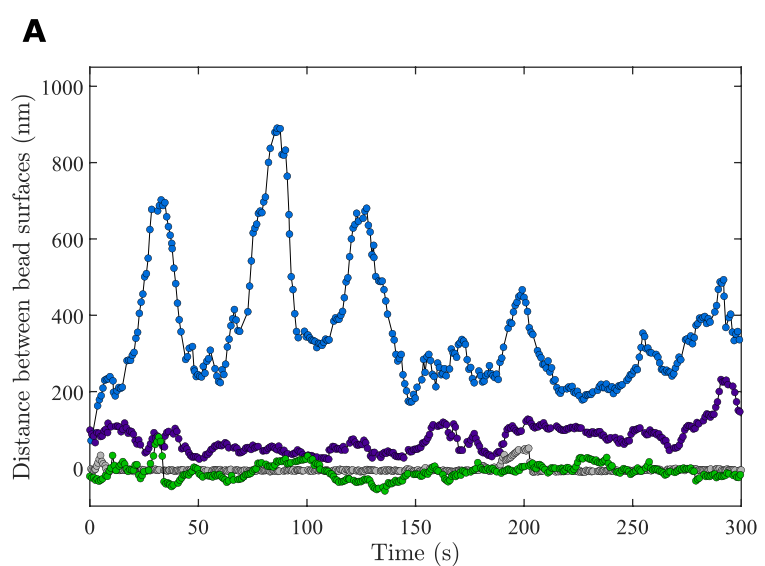

B

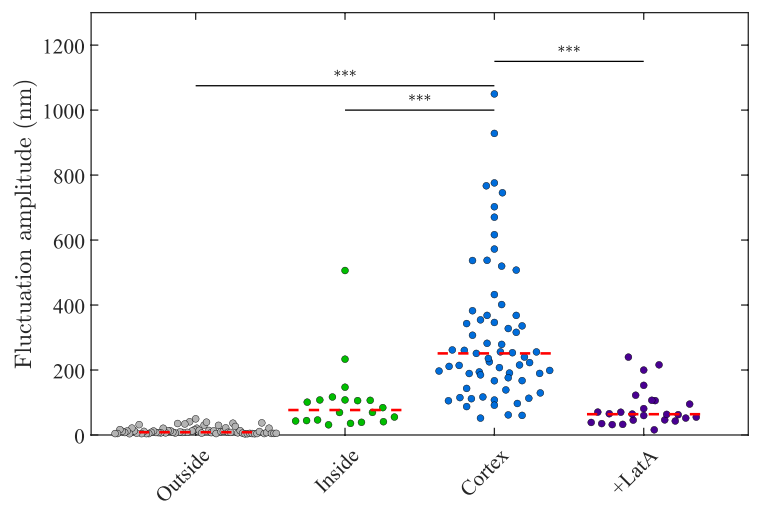

Fig. 2. Cortex thickness fluctuates in time. (A) Typical temporal evolution of the cortex thickness of control cells (blue) and LatA-treated cells (purple) compared to the temporal evolution of the distance between bead surfaces inside (green) or outside (gray) cells. Acquisition rate is $1.25 \mathrm{~Hz}$. Large fluctuations are visible in the measured cortex thickness of control cells (blue) but not in the other signals. (B) Amplitude of the cortex thickness fluctuations in control cells (blue, $n=67, N=10$ ) compared to the fluctuations of the distance between bead surfaces in LatA-treated cells (purple, $n=26, N=5$ ) and pairs of beads inside (green, $n=20, N=13$ ) or outside (gray, $n=69, N=2$ ) cells.

we observe large and fast fluctuations (several hundred nanometers in a few tens of seconds) for bead pairs pinching the cortex of a live cell. Most of these fluctuations are lost when cells are treated with LatA (Fig. 2A, quantified in Fig. 2B; see details in fig. S2A), showing that they are driven by the activity of the actin network. Internalized beads do not change the thickness or the fluctuations of the cortex, as these measurements do not vary with the number of beads ingested by the cells (fig. S2D). No periodicity is observed in the fluctuations of cortex thickness, as shown by the absence of peaks in the autocorrelation function (fig. S2F), and of any emerging frequency in the Fourier analysis (fig. S2E). Characteristic time scales for fluctuations can nonetheless be extracted, the median of their distribution being $20 \mathrm{~s}$. In some rare cases ( $n=4$ for control cells), the cortical layer is pinched at two different locations by two independent bead pairs (fig. S2G). No correlation between the signals of the two bead pairs is observed (fig. $\mathrm{S} 2 \mathrm{H}$ ), showing that thickness fluctuations are local rather than resulting from a global cell contraction. Cumulative distribution (fig. S2B) confirms the trend of large fluctuations in cortex thickness, and these fluctuations are strongly diminished 
when analyzing bead pairs inside cells or at the cortical layer of actin-depolymerized cells. This analysis further reveals an asymmetry in these active cortex fluctuations: Fluctuations associated with a thickness increase are larger than fluctuations associated with a thickness decrease (fig. S2C). The existence of this asymmetry implies that fluctuations that increase the thickness can be considered as "peaks," reflecting transient augmentation in the thickness of the cortical layer. We can thus extract a frequency by counting the number of peaks above a relevant threshold (see the Supplementary Materials). Control cell cortices exhibit, on average, 0.86 peaks per minute, while this number drops to 0.19 for cells treated with LatA (Fig. 3E). Together, these results show that the cortex is not a static structure with a constant thickness but is, on the contrary, a very dynamic object with large fluctuations in the direction perpendicular to the membrane. These fluctuations, nevertheless, remain below the resolution of classical imaging techniques, explaining why they had never been observed before. These measures therefore reveal a novel picture of the actin cortex as an unstable active layer that displays nonperiodical events of thinning and thickening.

\section{Cortex fluctuations depend on actin polymerization and actomyosin contractility}

While actin cortex thickness is mostly regulated by structural properties such as filament length (10), fast fluctuations more likely rely on active processes such as actin assembly and contractility. We thus investigate the role of actin nucleators, Arp2/3 and formin, and of the myosin II motors in this process. We treat the cells with small inhibitors after they had ingested the beads, as bead ingestion requires an active actin cytoskeleton.

Confocal imaging of LifeAct-GFP-expressing cells after drug treatment shows the expected effects of inhibition of actin nucleators on surface ruffles, formin inhibition having the most pronounced effect (Fig. 3A and fig. S3F). Actin nucleation impairment from formin or Arp2/3 inhibition leads to a moderate but significant reduction of cortex thickness and to a strong decrease of the amplitude of cortex thickness fluctuations (Fig. 3, C and D). This decrease is also visible in the cumulative distribution of thickness above the median (fig. S3A). In parallel with the decrease in amplitude, the number of peaks (larger than $100 \mathrm{~nm}$ ) observed per minute dropped to almost half of the control value (Fig. 3E). This tendency is more pronounced for the largest peaks (larger than $600 \mathrm{~nm}$ ) with a threefold reduction for Arp2/3 and formin inhibition (Fig. 3F). Formin inhibition, which almost completely abolished membrane ruffles visible on microscopy images, had a more limited effect on cortical thickness and fluctuations measured with the magnetic pincher. We performed a quantitative analysis of the fluctuation of the fluorescence signal in the cortex (fig. S4). It showed that fluctuations of the fluorescence signal are dominated by large actin structures, which are mostly suppressed by formin inhibition.

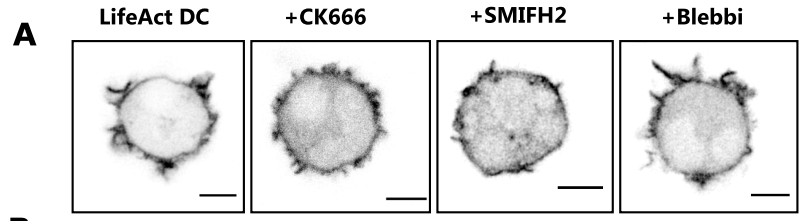

B

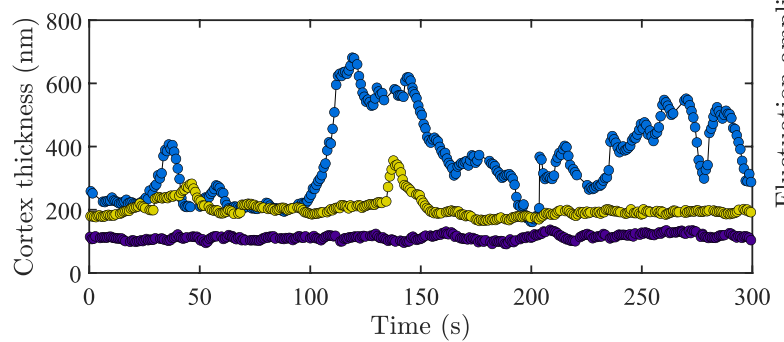

C
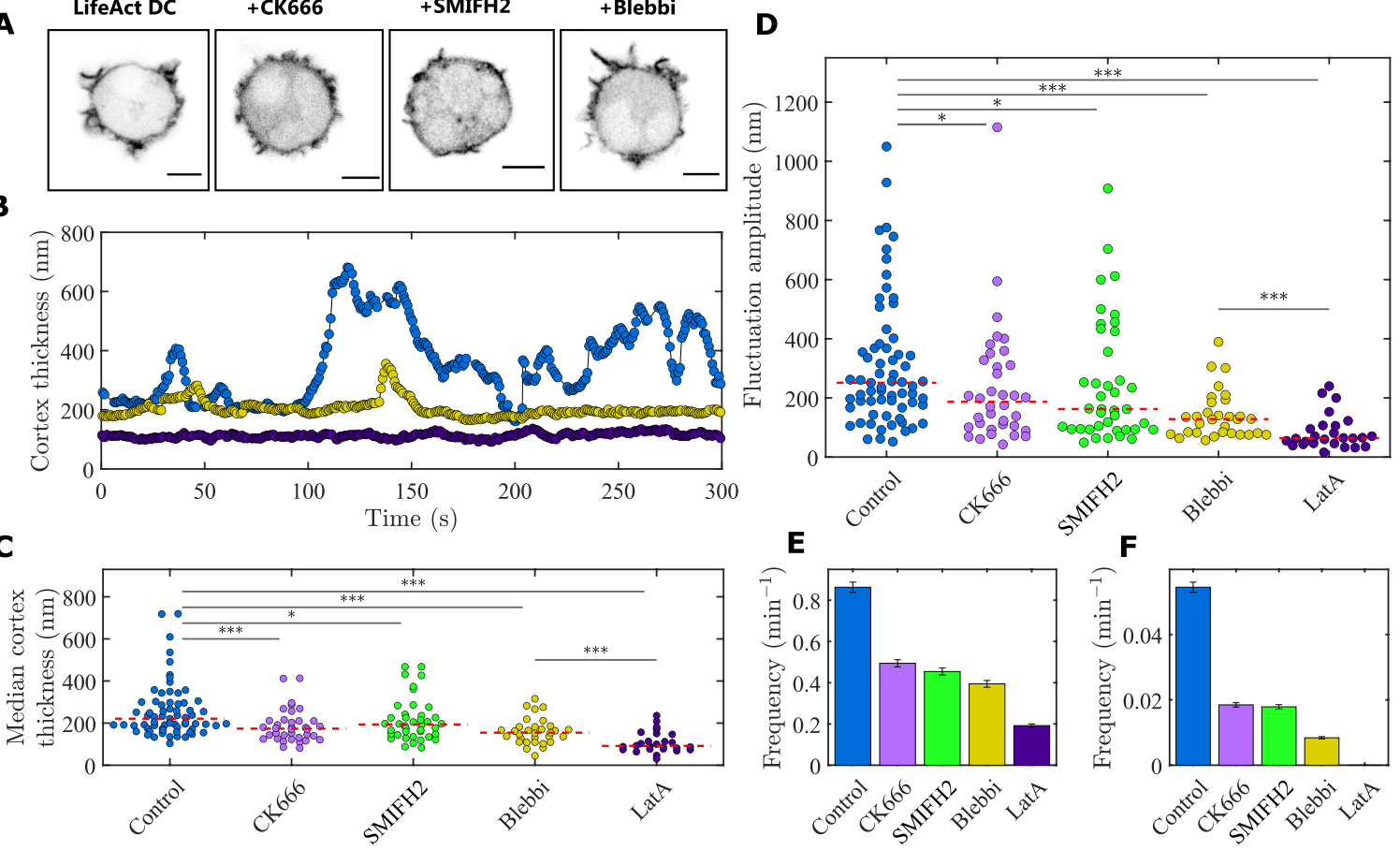

Fig. 3. Inhibition of myosin II affects cortex thickness and fluctuations more drastically than inhibition of nucleators. (A) Confocal imaging of actin in live LifeAct

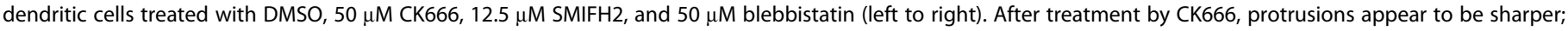
$\mathrm{SMIFH} 2$ treatment strongly reduces the number of protrusions; blebbistatin treatment slightly affects protrusion morphology. (B) Typical temporal evolution of the cortex thickness in control cells (blue), blebbistatin-treated cells (yellow), and LatA-treated cells (purple). (C) Median cortex thickness for control cells (blue, $n=67, N=10$ ) and cells treated with CK666 (light purple, $n=36, N=4$ ), SMIFH2 (green, $n=40, N=5$ ), blebbistatin (yellow, $n=31, N=5$ ), and LatA (dark purple, $n=26, N=5$ ). Control and LatA data are the same as in Fig. 1. (D) Amplitude of the cortex thickness fluctuations for control and treated cells [the same conditions as in (C)]. Myosin II inhibition has the strongest effect on fluctuations after LatA treatment but does not affect the morphology of the cell protrusions (A), leading to the conclusion that the measured fluctuations are not the signature of protrusion but rather fluctuations of the thickness of the underlying cortex. Control and LatA data are the same as in Fig. 2. (E) Frequency of peaks above $100 \mathrm{~nm}$ for control and treated cells. The reduced number of peak events in blebbistatin-treated cortices as well as with Arp2/3 and SMIFH2 inhibition is in agreement with the trend on fluctuation amplitude shown in (D). (F) Frequency of peaks larger than $600 \mathrm{~nm}$ for control and treated cells. 
Inhibition of myosin II motors using blebbistatin has, unexpectedly, a stronger effect than inhibition of actin nucleators on the properties of the cortex measured by the magnetic pincher. Cortical thickness is decreased by about one-third, and the amplitude of fluctuations is divided by two (Fig. 3, B to D), giving a cumulative probability of thickness variation close to the one of actin-depleted cells (fig. S3A). The frequency of actin-dependent protrusions is the lowest of all inhibition conditions, with 0.39 protrusions per minute (Fig. 3E) and a sixfold decrease in the frequency of peaks larger than $600 \mathrm{~nm}$ (Fig. 3F). As blebbistatin is phototoxic (26), we used the ROCK inhibitor Y27632 (Y27) to image the cells with a reduced motor activity. This small molecule reproduced the effect of blebbistatin on cortex thickness and associated fluctuations (fig. S3D). However, we observed only a limited effect on the ruffling activity of the dendritic cell membrane as compared to nontreated cells, with the quantitative fluorescence fluctuations analysis (figs. S3, E and F, and S4, C and E). Performing an analysis excluding regions of the cortex with large protrusions showed fluctuations in fluorescence intensity that depended more on myosin activity and less on formin (fig. S4, $\mathrm{D}$ and F) than the direct analysis (fig. S4, C and E). However, the measured reduction of fluctuations by the drugs was still different from the effects observed with the magnetic pincher for which myosin II inhibition had the largest consequences.

Together, the results on small-molecule inhibitors suggest that the submicrometer fluctuations in cortical thickness observed here do not result from the large surface ruffles but rather correspond to fluctuations in the thickness of the cortical layer itself. These submicrometer fluctuations are hard to detect from fluorescence microscopy images, whose signal is dominated by larger structures, more dependent on actin nucleators than on myosin II activity.

\section{A minimal physical description of the cortex recapitulating the effect of myosin II on thickness fluctuations}

Initial theoretical analysis of the actin cortex proposed that its thickness results from a balance between nucleation at the plasma membrane and bulk disassembly (27). A more recent analysis introduced the effect of modulation of filament length (10). However, none of these studies accounts for our observation of active submicrometer fluctuations of cortical thickness caused by myosin II activity. We thus turn to an extension of the minimal description in (24) that accounts for stress anisotropies (28). Here, the cortex is treated as an active viscous gel, with a constant influx of material at the membrane (representing polymerization) and homogeneous disassembly. The contractile property of the cortex is captured as an active stress that can be different in the directions tangential and perpendicular to the membrane due to the alignment of actin or myosin filaments (Fig. 4A). When the anisotropy in the contraction is low, a stable profile of actin density forming a compressed layer of constant thickness near the membrane emerges. However, when the anisotropy in the active stress exceeds a threshold so that the tangential stress is strong enough and stronger than the perpendicular one, the density profile becomes unstable. The cortex contracts laterally in an inhomogeneous manner, leading to local densification of actin. This induces the formation of peaks growing perpendicularly to the cortex plane. These peaks are not stable: They slide laterally and merge with each other, while new ones appear (Fig. 4B). The dynamical behavior of the fluctuations does not settle into a periodic state and is, on the contrary, aperiodic and chaotic, which matches our experimental observations. The amplitude of the fluctuations is of the same order of magnitude as the cortex thickness, and the frequency of those fluctuations is given by the characteristic depolymerization time of the cortex ( seconds), which also matches our experimental measures. The thickness fluctuations we observed in our experiments are thus a generic feature of actin networks assembling on a surface and exhibiting anisotropic contractility, which points to a major effect of contractility on cortex thickness stability.

To further analyze the analogy between our experimental results and the results of the theoretical analysis, we concentrate on the correlation between the cortical layer thickness and its fluctuation amplitude. In control cells, the amplitude of fluctuations in each single cell is strongly correlated with the thickness of the cortex (Fig. 4D, i). Although both cortical thickness and its fluctuation amplitude are reduced by the inhibition of actin nucleators (Fig. 4C, ii, and fig. S4B, i), this does not affect the correlation between the two (high correlation coefficient, low $P$ value, and slopes of the same order as for control cells, between 1.5 and 2; fig. S4, A and B). On the contrary, myosin II inhibition, which also reduces both the thickness of the cortex and the amplitude of fluctuations, has a strong effect on the correlation between the two parameters, with a lower correlation coefficient and a slope of only 0.67 (Fig. 4D, iii). In the case of LatA, the correlation completely disappears $(P=0.55)$. Coming back to the simulations, we vary the active stress and measure the cortex median thickness and its fluctuation amplitude in the same way as in the experiments (Fig. 4F). As only one length scale is present in the theoretical analysis, changing the resting size of the cortex by modifying the polymerization speed will affect in the same way both the cortex median thickness and the amplitude fluctuation. Thus, in both our experiments and theory, the contractile nature of the cortex controls the correlation between cortex thickness and its fluctuation amplitude. This finding suggests that the mechanism captured by the theory can explain the fluctuations in cortex thickness observed in live cells.

\section{DISCUSSION}

A large number of cell types display patterns of activity in the cell cortex in the form of polymerization waves, global contraction, or unorganized flares of activity $(29,30)$. These patterns are explained by a dual mechanism of activation and inhibition in regulatory pathways in interplay with the actin cytoskeleton (30-32). Myosin-dependent waves of Rho guanosine triphosphatase activity have been observed in adherent cells (33) and pulsatile patterns in embryos (34). Cortical flows could also entrain some larger structures embedded in the cortex and result in myosin-dependent local increase in the cortical thickness. Although we cannot rule out that these mechanisms contribute to the fluctuations of cortical thickness, the intrinsic dynamics of an active gel layer displayed in the presented model is sufficient to capture most of the characteristics of the fluctuations that we observed.

Collectively, our data propose a fundamentally new picture of the cell cortex in live cells as a fluctuating entity, whose thickness varies on a time scale of tens of seconds with spatial amplitude of hundreds of nanometers. This picture emerges through the new method presented here that provides a time-resolved measurement of the cortex thickness of live cells with an unprecedented spatial accuracy of a few tens of nanometers. We found the amplitude of thickness fluctuations to be comparable to the median value of the thickness. These results are not specific to primary mouse dendritic cells, as the cortex of Dictyostelium discoideum displays a similar behavior (see the Supplementary Materials and fig. S7). Fluctuations mostly result 
A

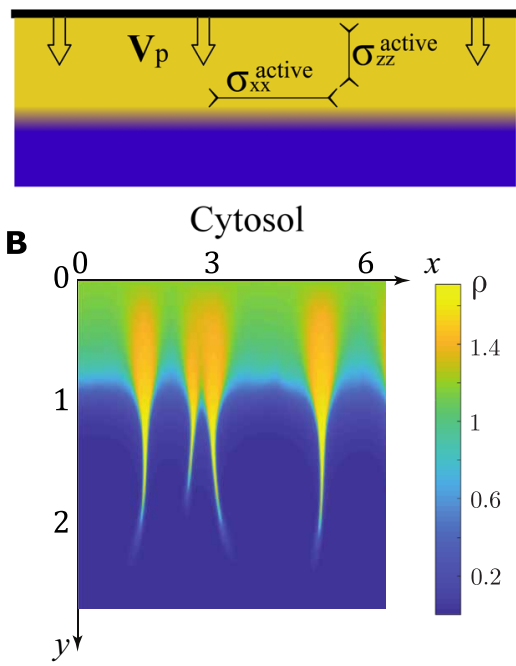

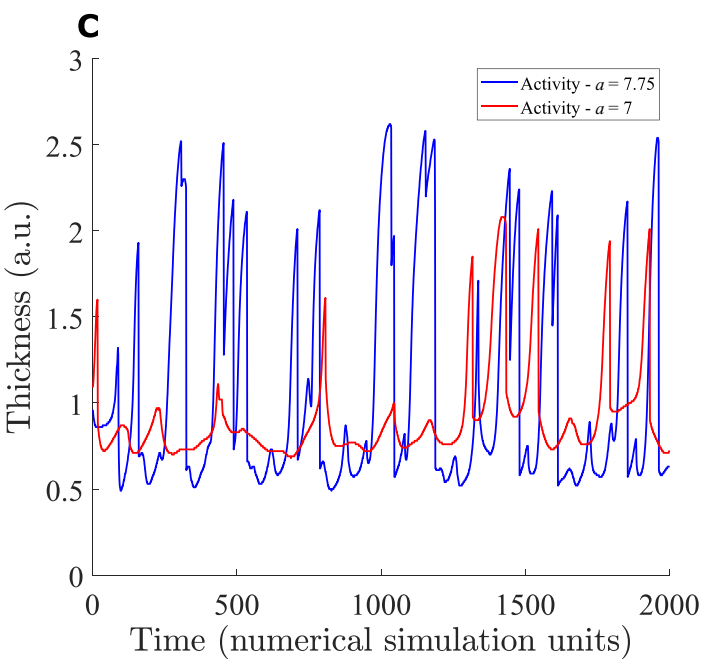

(ii)

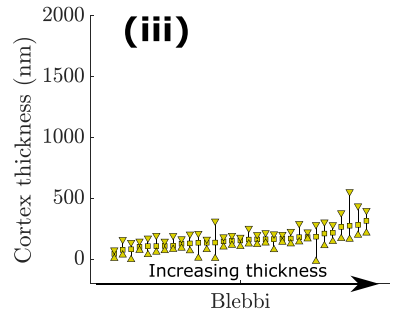

E

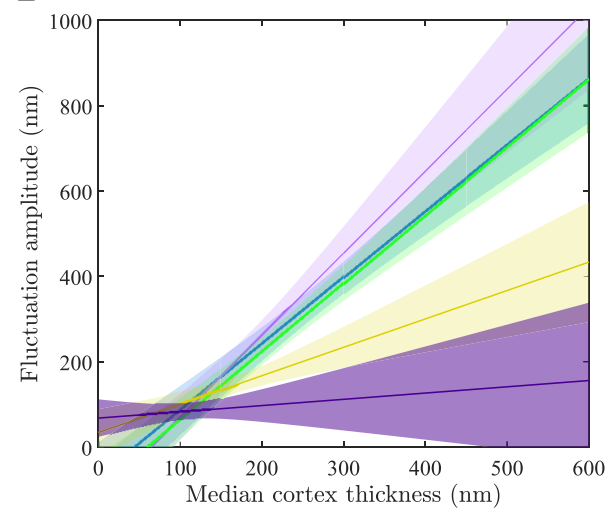

$\mathbf{F}$

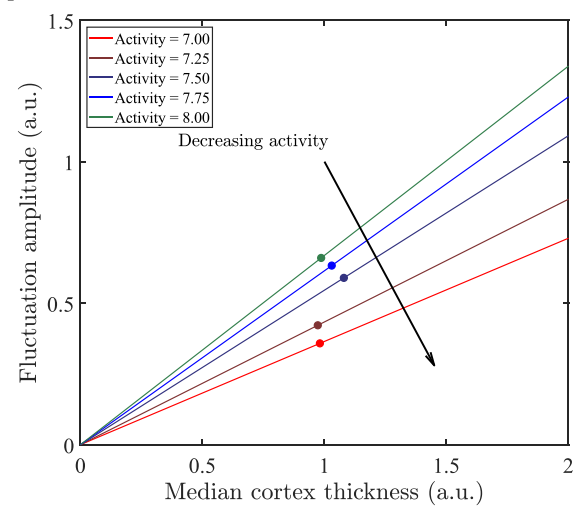

Fig. 4. A minimal theoretical description of the cortex captures the role of contractility in thickness fluctuations. (A) Illustration of the physical description of the cortex introducing the polymerization from the surface and the active stresses in the directions tangential $\left(\sigma_{\mathrm{xx}}\right)$ and perpendicular $\left(\sigma_{\mathrm{yy}}\right)$ to the membrane. (B) Snapshot of a numerical simulation using the cortex theoretical description illustrated in (A). The cortex locally deforms into protruding peaks because of the stress anisotropies in the actomyosin material. (C) Temporal evolution of the cortex thickness at one arbitrary spatial coordinate from a simulation at low contractile activity ( $a=7$, red) and at a higher contractile activity ( $a=7.75$, blue). The time unit is defined as the inverse of the gel's disassembly rate $k$ (see Materials and Methods). (D) Representation of the cortex median thickness and fluctuation amplitude from experiments on control cells (i), CK666-treated cells (ii), and blebbistatintreated cells (iii). Cells are sorted in ascending value of the median thickness (square). The length of the vertical line between the thickness first decile (upward triangle) and last decile (downward triangle) represents the fluctuation amplitude. The thickness fluctuations appear larger for larger cortex thicknesses for control cells but not for blebbistatin-treated cells. (E) Slopes from linear regression of fluctuation amplitudes as a function of cortex thickness from the experiments for control cells (blue) and cells treated with SMIFH2 (green, overlapping control), CK666 (light purple), blebbistatin (yellow), and LatA (dark purple). The colored area of each curve represents half the $95 \%$ confidence interval. The correlation of fluctuation amplitude with cortex thickness is markedly stronger for control and nucleator inhibition compared to myosin II inhibition. (F) Slopes for the theoretical relation between thickness and fluctuation amplitude calculated from simulations at varying activity (dots). a.u., arbitrary units. 
from the contractile activity of myosin II motors, which produces instabilities in the cortex, inducing large shifts perpendicular to the plasma membrane. They are intrinsically different from ruffles, which dominate the signal in fluorescence microscopy images. As our measure is local, it does not distinguish whether these shifts are due to bumps or wrinkles, but depicts the cortex as a dynamically embossed structure.

What are the putative functions of these cortical fluctuations that cells could modulate through the regulation of myosin II? One possible role could be to dynamically generate a rough plasma membrane surface that would augment the effective area of the cell surface and regulate membrane tension. Pulling membrane tethers from adherent fibroblasts revealed the existence of membrane reservoirs that depend on actin filaments (35). Gauthier et al. (36) proposed that these reservoirs come from submicrometer membrane wrinkles in the plasma membrane. The fluctuations in cortex thickness here reported could provide such a reservoir. Another possible function of a wrinkled or bumped cortex could be to provide dents on the outer surface of the cell to increase its friction with the substrate. Nonadherent cells can migrate faster than adherent cells, but the way forces are transmitted to the substrate in this migration mode is still mysterious. Having a dynamically rough surface could help dendritic cells generate propulsion forces in low adhesion environments. Last, the myosin-produced instabilities we evidenced give rise to fluctuations in thickness close to its median value. When these instabilities become too strong, the cortex could rupture, which could either induce local detachment of the plasma membrane called blebs or even lead to large-scale cell polarization $(19,34,37)$. This local membrane detachment from the cortex has been proposed to be responsible for initiating cell protrusions, including lamellipodia (38). It is thus tempting to speculate that the cortex fluctuations we observed with the magnetic pincher could serve to facilitate nucleation of cellular protrusions and more generally contribute to the fast cell shape changes observed in fast-migrating amoeboid cells such as dendritic cells.

Increasing the magnetic force between the beads provides a measurement of the cortex mechanical properties. A complete study of these properties is beyond the scope of the present paper, but we are able to provide an estimate of $7 \mathrm{kPa}$ for the elastic modulus of the cortex. No nonlinear behavior was detected even for cortices with up to $20 \%$ mean deformation (see the Supplementary Materials). This estimation of the elastic modulus of the cell cortex is similar to measurements obtained with atomic force microscopy using tips that are considered to probe the mechanics of the cortex itself (24). Although in vitro rheological studies on dilute entangled or crosslinked actin filament suspensions (39) report elastic modulus in the range of a few tens of pascals, it was shown that dense actin filament networks grown from a surface exhibit an elastic modulus of several kilopascals $(20,22,23)$. The modulus reported here is thus in accordance with these measures. The filament networks in these studies regularly exhibit nonlinear behaviors such as stress stiffening that are explained by either the large extension of entropic filaments in dilute networks (39) or the limited connectivity of branched networks (20). The absence of nonlinear behavior in the present work suggests that the filament network in the cortex is too dense and well connected to display these characteristics. The moderate hysteresis observed during compression and release is the signature of some viscoelastic behavior that can be due to cross-linkers and motors binding and unbinding to the actin filaments. Overall, the precise determination of both cortex thickness and material properties is a promising avenue to elucidate the quantitative contribution of the cortex mechanics to the global deformation of the cell in response to external mechanical stresses.

In conclusion, we believe that our observations not only provide measures of the physical properties of the cell cortex with unprecedented accuracy but also draw a novel picture for this subcellular entity as an active polymorphic layer dynamically thinned and thickened on a submicrometer scale as a result of actomyosin contractility. This could explain the propensity of the actin cortex to break upon activation of contractility, a phenomenon used by cells to polarize and move.

\section{MATERIALS AND METHODS \\ Materials}

The superparamagnetic beads (Dynabeads M-450) were purchased from Invitrogen (Carlsbad, CA, USA). Dimethyl sulfoxide (DMSO) was purchased from Sigma-Aldrich (St. Louis, MO, USA); SMIFH2, (R)-(+)-blebbistatin, CK666, and LatA were purchased from Tocris Bioscience (Bristol, UK).

\section{Magnetic setup}

The setup is mounted on an Axio A1 inverted microscope (Carl Zeiss, Germany) with an oil-immersion $100 \times$ objective [ 1.4 numerical aperture (NA)] mounted on a piezo-controlled translator (Physik Instrumente, Karlsruhe, Germany). The magnetic field is generated by two coaxial coils (SBEA, Vitry, France) with mu metal core (length, $40 \mathrm{~mm}$; diameter, 26 to $88 \mathrm{~mm}$; 750 spires). The coils are powered by a bipolar operational power supply amplifier 6A/36V (Kepco, Flushing, NY) controlled by a data acquisition module (National Instruments, Austin, TX). The maximum field generated is $100 \mathrm{mT}$ with a gradient less than $0.1 \mathrm{mT} \cdot \mathrm{mm}^{-1}$ over the sample. The chains of beads are formed with a constant field of $5 \mathrm{mT}$. Time-lapse images are recorded with an Orca Flash4 complementary metal-oxide semiconductor camera (Hamamatsu Photonics, Hamamatsu, Japan). No fluorescent images were taken during the magnetic pincher experiments to avoid phototoxicity when using sensitive drugs (e.g., blebbistatin). The setup was heated at $37^{\circ} \mathrm{C}$ using the box and the cube from Life Imaging Systems (Basel, Switzerland). The setup is controlled by a custom LabVIEW interface that ensures the synchronicity between piezo position, magnetic field imposition, and image acquisition.

\section{Cells}

Immature mouse bone marrow-derived dendritic cells were obtained by differentiation of bone marrow precursors for 10 to 11 days in dendritic cell medium [Iscove's modified Dulbecco's medium, fetal calf serum $(10 \%)$, glutamine $(20 \mathrm{mM})$, penicillin-streptomycin $\left(100 \mathrm{U} \mathrm{ml}^{-1}\right)$, and 2-mercaptoethanol $\left.(50 \mu \mathrm{m})\right]$ supplemented with granulocyte-macrophage colony-stimulating factor (50 $\mathrm{ng} \mathrm{ml}^{-1}$ )containing supernatant obtained from transfected J558 cells, as previously described (40).

\section{Mice}

LifeAct-GFP mice were a gift from M. Sixt (IST, Austria) (41) and bred in Institut Curie animal facilities. In general, 6- to 10-week-old mice were used as source for bone marrows to generate dendritic cells as described above. For animal care, the European and French National Regulation for the Protection of Vertebrate Animals used 
for Experimental and other Scientific Purposes (directive 2010/63; French decree 2013-118) was strictly followed. The present experiments, which used mouse strains displaying nonharmful phenotypes, did not require a project authorization and benefited from guidance of the Animal Welfare Body, Research Center, Institut Curie.

\section{Cell fluorescent imaging}

Spinning disc images were acquired on a Leica DMi8 microscope (Leica, Wetzlar, Germany) with an Orca Flash4 camera and a 100× objective. The setup was heated at $37^{\circ} \mathrm{C}$ using the box and the cube from Life Imaging Systems. Acquisition was done using the MetaMorph software. Confocal images were acquired using a laser scanning microscope (Leica TCS SP8) with a $40 \times$ oil immersion objective (1.4 NA), a HyD detector (Leica), and the LAS-AF software.

\section{Sample preparation}

Dynabeads M450 were washed three times in Milli-Q water and stored in dendritic cell medium. Cells were incubated at $5 \times$ $10^{5}$ cells $/ \mathrm{ml}$, with coated Dynabeads $\left(1: 1\right.$ ratio) for 1 hour at $37^{\circ} \mathrm{C}$ in dendritic cell medium supplemented with $20 \mathrm{mM}$ Hepes (SigmaAldrich). After 1 hour, $27 \pm 4 \%$ of the cells have ingested at least one bead. Observation was done in homemade polydimethylsiloxane (PDMS) chambers coated with $1 \%$ bovine serum albumin (Sigma-Aldrich). Cells were observed during a maximum of 1.5 hours with no measured differences between the beginning and the end of the experiment.

For TEM experiments, cells were plated on $\mu$-dishes with a glass bottom containing an imprinted $50-\mu \mathrm{m}$ cell location grid (Biovalley, ref. 81148). Cells were fixed in $2.5 \%$ glutaraldehyde in $0.1 \mathrm{M} \mathrm{Na}$ cacodylate buffer ( $\mathrm{pH}$ 7.4) for 1 hour, postfixed for 1 hour with $1 \%$ buffered osmium tetroxide, dehydrated in a graded series of ethanol solution, and then embedded in epoxy resin.

\section{Drug treatment}

CK666 and blebbistatin were used at $50 \mu \mathrm{M}$, SMIFH2 at $12.5 \mu \mathrm{M}$, and LatA at $500 \mathrm{nM}$, and the same quantity of DMSO was used in control experiments. PDMS chambers were preincubated with drugs 30 min before cell loading. Drugs were added to cell suspension at least 30 min before the beginning of the experiment.

\section{Data and statistical analysis}

Data relating to cortex thickness measurement come from 10 independent experiments for control, 4 independent experiments for CK666-treated cells, 5 independent experiments for SMIFH2treated cells, 5 independent experiments for blebbistatin-treated cells, and 5 independent experiments for LatA-treated cells. For "inside" and "outside" data (Fig. 1), the pairs of beads were observed when the wanted situation occurred during the previously described experiments. Data relating to cortex mechanical properties come from five independent experiments for control and one experiment for LatA-treated cells. Indentation curves of bare beads and serumcoated beads (Fig. 1) come from one experiment.

To determine the significance of the differences between distributions (Figs. 1 to 3 and figs. S2, S3, and S7), we used Wilcoxon rank sum test. In fig. S2D, the slope is represented as $\pm 95 \%$ confidence interval on the fit and the $P$ value is extracted directly from the fitting algorithm. In fig. S5, the $P$ value of the linear fits is extracted directly from the fitting algorithm. In fig. S8, the slope value is represented as $\pm 95 \%$ confidence interval on the fit.

\section{Determination of the bead distance}

Image analysis was carried out using Image (National Institutes of Health, USA) and Matlab (MathWorks, MA, USA). The in-plane position of each particle is determined by a weighted average of gray levels (fig. S5A), giving an accuracy of $2 \mathrm{~nm}$ as was done for in vitro work $(14,20)$. This accuracy can be obtained because the size of the Airy disc spans a few tens of pixels and because we tune the intensity of the incoming light to use the whole range of the 16-bit depth of the camera. A large number of information bits can thus be used to find the subpixel location of the center of the bead.

The position of the bead in the vertical direction is measured using the diffraction pattern on several images at different heights (42). Three images were recorded in quick succession (50-ms inter$\mathrm{val}$ ) at a height distance of $300 \mathrm{~nm}$ by moving the objective with a piezo-controlled translator. The diffraction patterns were compared to a reference (a depthograph), which was regularly updated. These depthographs are obtained by generating Z-stacks of immobilized beads and concatenating the central pixel lines of each bead image. Individual depthographs from independent beads are then averaged together to create the final reference.

A similar central pixel line is taken from a bead image on each of the three successive images and correlated onto the final depthograph (fig. S6B). The horizontal distance between the centers of two beads is taken as the average of these three measures. The precision on this procedure is estimated at $45 \mathrm{~nm}$ by cross-correlating independent depthographs and computing the average error. This leads to a precision of $7 \mathrm{~nm}$ on the $3 \mathrm{D}$ distance between the bead centers.

To ensure the quality of the tracking in the $\mathrm{Z}$ direction, data points are removed when a jump in $Z(>700 \mathrm{~nm})$ between two time points $(0.8 \mathrm{~s})$ is detected to avoid false measurements. Artefactual isolated points in the $3 \mathrm{D}$ distance curves were also removed (less than $0.05 \%$ for $\mathrm{Z}$ and $3 \mathrm{D}$ artefactual points). To ensure precision in the measurements of amplitude fluctuations of frequency of peaks, curves with too much removed data $(>50 \%)$ or that are too short $(<120 \mathrm{~s})$ were not considered for analysis ( $1 \%$ of total curves).

\section{Fluctuation and peak analysis}

The amplitude of fluctuations is computed as the difference between the 90th and 10th decile of a dataset. The asymmetry of a curve corresponds to $(a-b) /($ fluctuations amplitude) (fig. S2A).

To ensure the validity of the fluctuation quantification, we study the cumulative distribution of the measured thickness for being below a certain value (fig. S2B). The median thickness is removed from each cell, and the shown curve is the mean of the cumulative distribution of each cell. The same trend of large fluctuations for the cortical thickness layer remains apparent. These fluctuations are strongly diminished when looking at a pair of beads inside the cell or at the cortical layer of actin-depolymerized cells. An asymmetry is also apparent in the cortical layer fluctuation, with larger fluctuations increasing the size of the cortical layer and smaller fluctuations decreasing its size. The upper decile of thickness is 1.7 times more distant from the median than the lower decile (asymmetry $=27 \%$ ). This asymmetry is not present, with both beads inside the cells or with actin-depleted cells (fig. S2C).

To detect peaks, the signal is first smoothed using a SavitzkyGolay algorithm. The peaks are then detected as local maxima with a minimum prominence of $15 \mathrm{~nm}$. Before computing statistics, peaks with a gap in time or in height ( $>5 \mathrm{~s}$ or $>60 \%$ of prominence between two consecutive points) are removed from the data (less than 25\%). 
To avoid being dominated by noise, and to concentrate on actininduced fluctuations, we considered only the peaks above a threshold of $100 \mathrm{~nm}$ for further analysis (fig. S2I).

\section{Confocal image analysis}

To analyze actin intensity at the cell cortex, each cell is first "unfolded" using a homemade MATLAB algorithm. On each image of the video, the cell is identified with intensity thresholding and isolated from the rest of the image by putting all noncell pixel intensity to zero. The cell center is found using an Euclidian distance transform on the binarized cell image. Then, a coordinate system change is operated from cylindrical to Cartesian. As pixels have a size and do not land perfectly in an $(X, Y)$ grid from an $(R, \theta)$ grid, each "new pixel" intensity is computed as the weighted average of up to four old pixel intensities. The result is a video where the cell cortex appears linear (fig. S4A).

Cortex intensity value on each image is then taken as the maximum of vertical pixel line (fig. S4A, yellow lines and red dots) at 35 different positions along the cortex. From each position, a time curve is obtained (fig. S4B) and is analyzed in the same way as cortex thickness by measuring intensity median over time and intensity fluctuation between the first and last decile (fig. S4C). Spatial fluctuations (fig. S4E) are quantified as the standard deviation of the measured intensity distribution (35 points) on one image. In 10 -min videos, spatial fluctuations are measured on one image every $30 \mathrm{~s}$ to ensure independent measurements through renewal of the actin cortex.

To assess the influence of protrusions in these intensity measurements, the same analysis was conducted after removing measurement points that where in protrusions (e.g., position 1 in fig. S4A). These points were identified by quantifying the number of nonzero pixels that extend from the maximum toward the exterior of the cell (fig. S4A, cyan lines on positions 2 and 4).

\section{Theoretical description of the cortex}

For the theoretical analysis, we use the following physical description of the cortex as an active gel leading (28). We consider that the cortex is growing into the half-space $z \geq 0$ at the surface $(x, y, z=0)$ because of polymerization. We note that $\rho$ is the density of the actin gel and $\mathrm{v}=\left(\mathrm{v}_{x}, \mathrm{v}_{y}, \mathrm{v}_{z}\right)$ is the velocity field. For the sake of simplicity, we assume invariance along the $y$ direction and $\mathrm{v}_{y}=0$. Three equations determine the temporal evolution of the cortex

$$
\begin{gathered}
\partial_{t} \rho+\partial_{x}\left(\rho \mathrm{v}_{x}\right)+\partial_{z}\left(\rho \mathrm{v}_{z}\right)=-k \rho \\
\eta\left[2 \partial_{x x} \mathrm{v}_{x}+\partial_{x z} \mathrm{v}_{z}+\partial_{z z} \mathrm{v}_{x}\right]=\partial_{x} \Pi_{x}(\rho) \\
\eta\left[2 \partial_{z z} v_{z}+\partial_{x z} \mathrm{v}_{x}+\partial_{x x} \mathrm{v}_{z}\right]=\partial_{z} \Pi_{z}(\rho)
\end{gathered}
$$

The first equation accounts for mass conservation and the two last ones for force balance. Here, $k$ denotes the gel's disassembly rate, $\eta$ is the viscosity, and $\Pi_{x, z}$ are the components of the nonviscous contribution to the total stress in the gel. The latter has two contributions: an effective hydrostatic pressure and the contractile stress generated by active processes in the gel. Both components depend on the gel density, and we write $(27,28)$

$$
\Pi_{x, z}=-a_{x, z} \rho^{3}+b \rho^{4}
$$

with $b>0$ accounting for positive hydrostatic pressure and $a_{x, z}>0$, which reflects contractility of the active stress component.

These equations are complemented by the boundary conditions $\mathrm{v}_{z}(z=0)=\mathrm{v}_{p}$ and $\mathrm{v}_{\mathrm{x}}(z=0)=0$, where $\mathrm{v}_{p}$ denotes the polymerization speed. See (28) for a discussion about more general boundary conditions including friction between the cortex and the membrane. Note that the sole length scale of this description is $l=v_{p} k^{-1}$. This implies that for a given value of $a_{x, z} / \eta$ and $b / \eta$, the amplitude of the fluctuations and the median thickness are proportional.

The set of previous equations generates spontaneously chaotic protrusions from the cortical layer if the active parameter $a_{x}$ is large enough (28). Figure 4 (B, C, and F) is obtained by solving numerically the above equations. To this end, we used a discrete Euler scheme: In each time step, we first determine the velocity field through the force balance equations, where we use Fourier decomposition along $x$ and finite differences along $z$. We then update the density. The contribution of $\partial_{z}\left(\rho v_{z}\right)$ is obtained by an upwind finite-difference scheme in real space. To improve the stability of the scheme, we have added a small diffusion term with diffusion constant $D=10^{-3}$ to the mass conservation equation. In all simulations, we use $\Delta x=0.004, \Delta z=$ 0.007 , and $\Delta t=0.0005$. Last, we define the thickness of the cortex by the smallest $z$ value for which the actin density dropped to half of its value at $z=0$. Note that choosing another criterium does not affect the qualitative behavior of our results.

\section{SUPPLEMENTARY MATERIALS}

Supplementary material for this article is available at http://advances.sciencemag.org/cgi/ content/full/7/27/eabe3640/DC1

View/request a protocol for this paper from Bio-protocol.

\section{REFERENCES AND NOTES}

1. T. D. Pollard, J. A. Cooper, Actin, a central player in cell shape and movement. Science $\mathbf{3 2 6}$ 1208-1212 (2009).

2. P. Chugh, E. K. Paluch, The actin cortex at a glance. J. Cell Sci. 131, jcs186254 (2018).

3. G. Salbreux, G. Charras, E. Paluch, Actin cortex mechanics and cellular morphogenesis. Trends Cell Biol. 22, 536-545 (2012).

4. A. Diz-Muñoz, O. D. Weiner, D. A. Fletcher, In pursuit of the mechanics that shape cell surfaces. Nat. Phys. 14, 648-652 (2018).

5. M. Fritzsche, C. Erlenkämper, E. Moeendarbary, G. Charras, K. Kruse, Actin kinetics shapes cortical network structure and mechanics. Sci. Adv. 2, e1501337 (2016).

6. M. Bovellan, Y. Romeo, M. Biro, A. Boden, P. Chugh, A. Yonis, M. Vaghela, M. Fritzsche, D. Moulding, R. Thorogate, A. Jégou, A. J. Thrasher, G. Romet-Lemonne, P. P. Roux, E. K. Paluch, G. Charras, Cellular control of cortical actin nucleation. Curr. Biol. 24, 1628-1635 (2014).

7. M. Biro, Y. Romeo, S. Kroschwald, M. Bovellan, A. Boden, J. Tcherkezian, P. P. Roux, G. Charras, E. K. Paluch, Cell cortex composition and homeostasis resolved by integrating proteomics and quantitative imaging. Cytoskeleton 70, 741-754 (2013).

8. T. M. Svitkina, Actin cell cortex: Structure and molecular organization. Trends Cell Biol. 30, 556-565 (2020).

9. A. G. Clark, K. Dierkes, E. K. Paluch, Monitoring actin cortex thickness in live cells. Biophys. J. 105, 570-580 (2013).

10. P. Chugh, A. G. Clark, M. B. Smith, D. A. D. Cassani, K. Dierkes, A. Ragab, P. P. Roux, G. Charras, G. Salbreux, E. K. Paluch, Actin cortex architecture regulates cell surface tension. Nat. Cell Biol. 19, 689-697 (2017).

11. M. P. Clausen, H. Colin-York, F. Schneider, C. Eggeling, M. Fritzsche, Dissecting the actin cortex density and membrane-cortex distance in living cells by super-resolution microscopy. J. Phys. D Appl. Phys. 50, 064002 (2017).

12. R. Vargas-Pinto, H. Gong, A. Vahabikashi, M. Johnson, The effect of the endothelial cell cortex on atomic force microscopy measurements. Biophys. J. 105, 300-309 (2013).

13. B. Fabry, G. N. Maksym, J. P. Butler, M. Glogauer, D. Navajas, J. J. Fredberg, Scaling the microrheology of living cells. Phys. Rev. Lett. 87, 148102 (2001).

14. T. Pujol, O. du Roure, M. Fermigier, J. Heuvingh, Impact of branching on the elasticity of actin networks. Proc. Natl. Acad. Sci. U.S.A. 109, 10364-10369 (2012). 
15. R. Belbahri, J. Planade, M. B. Sanders, A. Guillotin, O. du Roure, A. Michelot, J. Heuvingh, Mechanical stiffness of reconstituted actin patches correlates tightly with endocytosis efficiency. PLOS Biol. 17, e3000500 (2019).

16. J. Renkawitz, K. Schumann, M. Weber, T. Lämmermann, H. Pflicke, M. Piel, J. Polleux, J. P. Spatz, M. Sixt, Adaptive force transmission in amoeboid cell migration. Nat. Cell Biol. 11, 1438-1443 (2009).

17. T. Lämmermann, B. L. Bader, S. J. Monkley, T. Worbs, R. Wedlich-Söldner, K. Hirsch, M. Keller, R. Förster, D. R. Critchley, R. Fässler, M. Sixt, Rapid leukocyte migration by integrin-independent flowing and squeezing. Nature 453, 51-55 (2008).

18. P. Vargas, P. Maiuri, M. Bretou, P. J. Sáez, P. Pierobon, M. Maurin, M. Chabaud, D. Lankar, D. Obino, E. Terriac, M. Raab, H.-R. Thiam, T. Brocker, S. M. Kitchen-Goosen, A. S. Alberts, P. Sunareni, S. Xia, R. Li, R. Voituriez, M. Piel, A.-M. Lennon-Duménil, Innate control of actin nucleation determines two distinct migration behaviours in dendritic cells. Nat. Cell Biol. 18, 43-53 (2016)

19. Y.-J. Liu, M. Le Berre, F. Lautenschlaeger, P. Maiuri, A. Callan-Jones, M. Heuzé, T. Takaki, R. Voituriez, M. Piel, Confinement and low adhesion induce fast amoeboid migration of slow mesenchymal cells. Cell 160, 659-672 (2015).

20. P. Bauër, J. Tavacoli, T. Pujol, J. Planade, J. Heuvingh, O. Du Roure, A new method to measure mechanics and dynamic assembly of branched actin networks. Sci. Rep. 7 15688 (2017).

21. M. P. Serres, M. Samwer, B. A. Truong Quang, G. Lavoie, U. Perera, D. Görlich, G. Charras, M. Petronczki, P. P. Roux, E. K. Paluch, F-actin interactome reveals vimentin as a key regulator of actin organization and cell mechanics in mitosis. Dev. Cell 52, 210-222.e7 (2020).

22. Y. Marcy, J. Prost, M.-F. Carlier, C. Sykes, Forces generated during actin-based propulsion: A direct measurement by micromanipulation. Proc. Natl. Acad. Sci. U.S.A. 101, 5992-5997 (2004).

23. P. Bieling, T.-D. Li, J. Weichsel, R. McGorty, P. Jreij, B. Huang, D. A. Fletcher, R. D. Mullins, Force feedback controls motor activity and mechanical properties of self-assembling branched actin networks. Cell 164, 115-127 (2016).

24. P.-H. Wu, D. R.-B. Aroush, A. Asnacios, W.-C. Chen, M. E. Dokukin, B. L. Doss, P. Durand-Smet, A. Ekpenyong, J. Guck, N. V. Guz, P. A. Janmey, J. S. H. Lee, N. M. Moore, A. Ott, Y.-C. Poh, R. Ros, M. Sander, I. Sokolov, J. R. Staunton, N. Wang, G. Whyte, D. Wirtz, A comparison of methods to assess cell mechanical properties. Nat. Methods 15, 491-498 (2018).

25. M. Guo, A. J. Ehrlicher, M. H. Jensen, M. Renz, J. R. Moore, R. D. Goldman, J. Lippincott-Schwartz F. C. Mackintosh, D. A. Weitz, Probing the stochastic, motor-driven properties of the cytoplasm using force spectrum microscopy. Cell 158, 822-832 (2014).

26. A. Mikulich, S. Kavaliauskiene, P. Juzenas, Blebbistatin, a myosin inhibitor, is phototoxic to human cancer cells under exposure to blue light. Biochim. Biophys. Acta 1820, 870-877 (2012).

27. J.-F. Joanny, K. Kruse, J. Prost, S. Ramaswamy, The actin cortex as an active wetting layer Active Matter. Guest editors: Ramin Golestarian, Sriram Ramaswamy. Eur. Phys. J. E. 36, 52 (2013).

28. N. Levernier, K. Kruse, Spontaneous formation of chaotic protrusions in a polymerizing active gel layer. New J. Phys. 22, 013003 (2020)

29. Y. Yang, M. Wu, Rhythmicity and waves in the cortex of single cells. Philos. Trans. R. Soc. B Biol. Sci. 373, 20170116 (2018)

30. W. M. Bement, M. Leda, A. M. Moe, A. M. Kita, M. E. Larson, A. E. Golding, C. Pfeut, K.-C. Su, A. L. Miller, A. B. Goryachev, G. von Dassow, Activator-inhibitor coupling between Rho signaling and actin assembly make the cell cortex an excitable medium. Nat. Cell Biol. 17, 1471-1483 (2015).

31. P. N. Devreotes, S. Bhattacharya, M. Edwards, P. A. Iglesias, T. Lampert, Y. Miao, Excitable signal transduction networks in directed cell migration. Annu. Rev. Cell Dev. Biol. 33 103-125 (2017).

32. D. S. Pal, X. Li, T. Banerjee, Y. Miao, P. N. Devreotes, The excitable signal transduction networks: Movers and shapers of eukaryotic cell migration. Int. J. Dev. Biol. 63, 407-416 (2019).

33. M. Graessl, J. Koch, A. Calderon, D. Kamps, S. Banerjee, T. Mazel, N. Schulze, J. K. Jungkurth, R. Patwardhan, D. Solouk, N. Hampe, B. Hoffmann, L. Dehmelt, P. Nalbant,
An excitable Rho GTPase signaling network generates dynamic subcellular contraction patterns. J. Cell Biol. 216, 4271-4285 (2017).

34. M. Nishikawa, S. R. Naganathan, F. Jülicher, S. W. Grill, Controlling contractile instabilities in the actomyosin cortex. elife 6, e19595 (2017).

35. D. Raucher, M. P. Sheetz, Membrane expansion increases endocytosis rate during mitosis. J. Cell Biol. 144, 497-506 (1999).

36. N. C. Gauthier, T. A. Masters, M. P. Sheetz, Mechanical feedback between membrane tension and dynamics. Trends Cell Biol. 22, 527-535 (2012).

37. V. Ruprecht, S. Wieser, A. Callan-Jones, M. Smutny, H. Morita, K. Sako, V. Barone, M. Ritsch-Marte, M. Sixt, R. Voituriez, C. P. Heisenberg, Cortical contractility triggers a stochastic switch to fast amoeboid cell motility. Cell 160, 673-685 (2015).

38. E. S. Welf, C. E. Miles, J. Huh, M. K. Driscoll, T. Isogai, J. Noh, A. D. Weems, J. Chi, T. Pohlkamp, K. Dean, R. Fiolka, A. Mogilner, G. Danuser, A unified role for membranecortex detachment during cell protrusion initiation. bioRxiv 696211 [Preprint]. 9 July 2019. https://doi.org/10.1101/696211.

39. M. L. Gardel, J. H. Shin, F. C. MacKintosh, L. Mahadevan, P. Matsudaira, D. A. Weitz, Elastic behavior of cross-linked and bundled actin networks. Science 304, 1301-1305 (2004).

40. H.-R. Thiam, P. Vargas, N. Carpi, C. L. Crespo, M. Raab, E. Terriac, M. C. King, J. Jacobelli, A. S. Alberts, T. Stradal, A.-M. Lennon-Dumenil, M. Piel, Perinuclear Arp2/3-driven actin polymerization enables nuclear deformation to facilitate cell migration through complex environments. Nat. Commun. 7, 10997 (2016).

41. J. Riedl, K. C. Flynn, A. Raducanu, F. Gärtner, G. Beck, M. Bösl, F. Bradke, S. Massberg, A. Aszodi, M. Sixt, R. Wedlich-Söldner, Lifeact mice for studying F-actin dynamics. Nat. Methods 7, 168-169 (2010).

42. C. Gosse, V. Croquette, Magnetic Tweezers: Micromanipulation and force measurement at the molecular level. Biophys. J. 82, 3314-3329 (2002).

43. L. D. Landau, E. M. Lifshitz, Theory of Elasticity (Pergamon Press, 1960), vol. 64, pp. 176-177.

44. R. S. Chadwick, Axisymmetric indentation of a thin incompressible elastic layer. SIAM J. Appl. Math. 62, 1520-1530 (2002).

Acknowledgments: We thank P. Belska for preliminary experiments on the magnetic pincher R. Voituriez for useful discussions; X. Benoit Gonin and O. Brouard for technical support on the setup; A. Mathur, M. Deygas, H. Moreau, D. Sanseau, G. Delgado, and Z. Alraies for additional cell culture; and N. Srivastava for help on $D$. discoideum culture and handling. Funding: This work was supported by the Institut Pierre-Gilles de Gennes-IPGG (Equipement d'Excellence, "Investissements d'avenir," program ANR-10-EQPX-34 and Laboratoire d'Excellence, "Investissements d'avenir" program ANR-10-IDEX-0001-02 PSL and ANR-10-LABX-31). V.L. was supported by Inserm (16DOC011UPDE / FDV 2016001) and by ANR grant (ANR-19CE13-0030-01) to M.P. K.K. was supported by SNSF grant (205321_17599). Author contributions: O.d.R., M.P., and J.H. designed the research; V.L., O.d.R., and J.H. developed the cortex pinching experiments; V.L. carried out all the cortex pinching experiments and analyzed the resulting data; L.B. and P.J.S. performed differentiation and culture of dendritic cells under the supervision of P.V.; J.P. carried out all confocal imaging experiments; M.S.R. performed electronic microscopy experiments; V.L. performed live-cell spinning disc imaging; V.L., L.B., and P.J.S. carried out and analyzed immunofluorescence experiments; K.K. and N.L. developed the theoretical analysis; N.L. performed and analyzed cortex simulations; V.L., O.d.R., M.P., and J.H. interpreted experimental data with the contribution of P.V. and A.-M.L.-D.; V.L., O.d.R., M.P., and J.H. wrote the manuscript. Competing interests: The authors declare that they have no competing interests. Data and materials availability: All data needed to evaluate the conclusions in the paper are present in the paper and/or the Supplementary Materials.

Submitted 17 August 2020

Accepted 20 May 2021

Published 2 July 2021

$10.1126 /$ sciadv.abe 3640

Citation: V. Laplaud, N. Levernier, J. Pineau, M. S. Roman, L. Barbier, P. J. Sáez, A.-M. Lennon-Duménil P. Vargas, K. Kruse, O. du Roure, M. Piel, J. Heuvingh, Pinching the cortex of live cells reveals thickness instabilities caused by myosin II motors. Sci. Adv. 7, eabe3640 (2021). 


\section{ScienceAdvances}

\section{Pinching the cortex of live cells reveals thickness instabilities caused by myosin II motors}

Valentin LaplaudNicolas LevernierJudith PineauMabel San RomanLucie BarbierPablo J. SáezAna-Maria LennonDuménilPablo VargasKarsten KruseOlivia du RoureMatthieu PielJulien Heuvingh

Sci. Adv., 7 (27), eabe3640. • DOI: 10.1126/sciadv.abe3640

View the article online

https://www.science.org/doi/10.1126/sciadv.abe3640

Permissions

https://www.science.org/help/reprints-and-permissions 